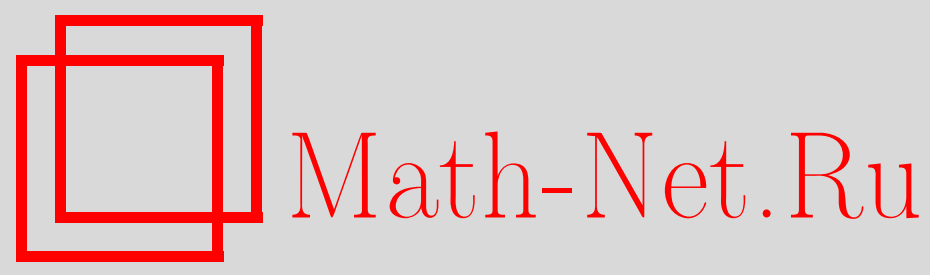

В. А. Краснов, Об алгебраических когомологиях вещественных алгебраических $M$-многообразий, Матем. заметки, 2004, том 76, выпуск 6, 854-867

DOI: https://doi.org/10.4213/mzm157

Использование Общероссийского математического портала Math-Net.Ru подразумевает, что вы прочитали и согласны с пользовательским соглашением http://www.mathnet.ru/rus/agreement

Параметры загрузки:

IP : 54.198 .55 .26

26 апреля 2023 г., $07: 16: 30$

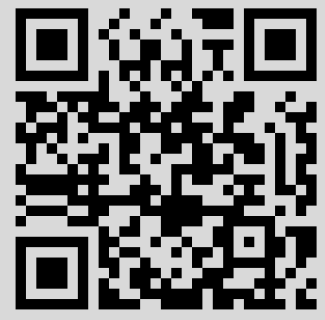




\section{ОБ АЛГЕБРАИЧЕСКИХ КОГОМОЛОГИЯХ ВЕЩЕСТВЕННЫХ АЛГЕБРАИЧЕСКИХ $M$-МНОГООБРАЗИЙ}

\section{В. А. Краснов}

Для вещественного алгебраического $M$-многообразия $X$ рассматривается канонический гомоморфизм алгебраической группы когомологий множества вещественных точек в алгебраическую группу когомологий множества комплексных точек

$$
\varrho_{k}: H_{\mathrm{alg}}^{k}(X(\mathbb{R}), \mathbb{Z} / 2) \rightarrow H_{\mathrm{alg}}^{2 k}(X(\mathbb{C}), \mathbb{Z} / 2) .
$$

Этот гомоморфизм согласован с отображениями цикла. Производятся оценки размерности ядра данного гомоморфизма.

Библиограффия: 8 названий.

Введение. Пусть далее $X-n$-мерное неособое проективное вещественное алгебраическое многообразие, т.е. $X$ - такая схема над $\mathbb{R}$, что произведение $X_{\mathbb{C}}=X \otimes \mathbb{C}$ является $n$-мерным неособым проективным комплексным алгебраическим многообразием. Множество комплексных точек $X(\mathbb{C})$ является компактньм комплексным $n$-мерным многообразием, а множество вещественных точек $X(\mathbb{R})$, если оно непустое, является $n$-мерньп компактньм дифференцируемым многообразием (может быть, несвязньп). Будем предполагать, что $X$ является $M$-многообразием, тогда множество $X(\mathbb{R})$ непустое. Напомним, что $X$ назьвается $M$-многообразием, если неравенство Гарнака-Тома

$$
\operatorname{dim}_{\mathbb{Z} / 2} H^{*}(X(\mathbb{R}), \mathbb{Z} / 2) \leqslant \operatorname{dim}_{\mathbb{Z} / 2} H^{*}(X(\mathbb{C}), \mathbb{Z} / 2)
$$

становится равенством. Пусть $A^{*}\left(X_{\mathbb{C}}\right)$ - кольцо Чжоу многообразия $X_{\mathbb{C}}$, тогда определено комплексное отображение цикла

$$
\operatorname{cl}_{\mathbb{C}}: A^{*}\left(X_{\mathbb{C}}\right) \rightarrow H^{*}(X(\mathbb{C}), \mathbb{Z} / 2)
$$

которое циклу $Z \in A^{q}\left(X_{\mathbb{C}}\right)$ сопоставляет класс когомологий

$$
[Z(\mathbb{C})]^{*} \in H^{2 q}(X(\mathbb{C}), \mathbb{Z} / 2) .
$$

Образ этого отображения обозначим через $H_{\mathrm{alg}}^{*}(X(\mathbb{C}), \mathbb{Z} / 2)$ и назовем комплексной алгебраической группой когомологий. Кроме комплексного отображения цикла определено вещественное отображение цикла

$$
\mathrm{cl}_{\mathbb{R}}: A^{*}(X) \rightarrow H^{*}(X(\mathbb{R}), \mathbb{Z} / 2),
$$

Работа выполнена при финансовой поддержке фонда INTAS, грант INTAS-OPEN-2000-269. 
где $A^{*}(X)$ - кольцо Чжоу многообразия $X$, которое циклу $Z \in A^{q}(X)$ сопоставляет класс когомологий

$$
[Z(\mathbb{R})]^{*} \in H^{q}(X(\mathbb{R}), \mathbb{Z} / 2) .
$$

Образ этого отображения цикла обозначим через $H_{\mathrm{alg}}^{*}\left(X(\mathbb{R}), \mathbb{F}_{2}\right)$ и назовем вещественной алгебраической группой когомологий. Операция комплексификации для циклов определяет гомоморфизм колец Чжоу $A^{*}(X) \rightarrow A^{*}\left(X_{\mathbb{C}}\right)$, композицию его с отображением цикла $\mathrm{cl}_{\mathbb{C}}$ будем также обозначать через

$$
\mathrm{cl}_{\mathbb{C}}: A^{*}(X) \rightarrow H^{*}(X(\mathbb{C}), \mathbb{Z} / 2) .
$$

Заметим, что циклу $Z \in A^{q}(X)$ это отображение сопоставляет класс когомологий

$$
[Z(\mathbb{C})]^{*} \in H^{q}(X(\mathbb{C}), \mathbb{Z} / 2) .
$$

Для $M$-многообразия $X$ справедливо следующее

УТВЕРЖДЕНИЕ (см. [1]). Если $\mathrm{cl}_{\mathbb{R}}(Z)=0$, mo maкже $\mathrm{cl}_{\mathbb{C}}(Z)=0$.

Поэтому для $M$-многообразия однозначно определен гомоморфизм

$$
\varrho_{*}: H_{\mathrm{alg}}^{*}(X(\mathbb{R}), \mathbb{Z} / 2) \rightarrow H_{\mathrm{alg}}^{*}(X(\mathbb{C}), \mathbb{Z} / 2),
$$

для которого коммутативна диаграмма

$$
H_{\text {alg }}^{*}(X(\mathbb{R}), \mathbb{Z} / 2) \stackrel{\varrho_{*}}{\longrightarrow} H_{\text {alg }}^{*}(X(\mathbb{C}), \mathbb{Z} / 2)
$$

Главная цель этой работы - изучить гомоморфизм $\varrho_{*}$, в частности, оценить размерность его ядра (имеется ввиду размерность над полем $\mathbb{Z} / 2$ ). Приведем пример такой оценки, которая доказьвается очень просто. Для этого обозначим факторгрушу

$$
H^{n-k}(X(\mathbb{R}), \mathbb{Z} / 2) / H_{\text {alg }}^{n-k}(X(\mathbb{R}), \mathbb{Z} / 2)
$$

через $H_{\mathrm{tr}}^{n-k}(X(\mathbb{R}), \mathbb{Z} / 2)$ и назовем ее трансиендентной группой когомологий. Тогда выполняется неравенство

$$
\operatorname{dim} \operatorname{ker} \varrho_{k} \leqslant \operatorname{dim} H_{\mathrm{tr}}^{n-k}(X(\mathbb{R}), \mathbb{Z} / 2) .
$$

Для доказательства этой оценки достаточно установить следующее утверждение: $е с л и$ $Z_{1} \in A^{k}(X)$ и $Z_{2} \in A^{n-k}(X)$, причем $\mathrm{cl}_{\mathbb{R}}\left(Z_{1}\right) \in \operatorname{ker} \varrho_{k}$, то классь когомологий $\operatorname{cl}_{\mathbb{R}}\left(Z_{1}\right), \mathrm{cl}_{\mathbb{R}}\left(Z_{2}\right)$ ортогональны относительно спаривания групп когомологий

$$
H^{k}(X(\mathbb{R}), \mathbb{Z} / 2), \quad H^{n-k}(X(\mathbb{R}), \mathbb{Z} / 2)
$$

c помощью индекса пересечения. А это утверждение вытекает из равенства индексов пересечения со значениями в $\mathbb{Z} / 2$

$$
\left(Z_{1}(\mathbb{R}), Z_{2}(\mathbb{R})\right)=\left(Z_{1}(\mathbb{C}), Z_{2}(\mathbb{C})\right) .
$$


1. Старые и новые теоремы. Сформулируем сначала некоторые известные результаты про гомоморфизм $\varrho_{*}$. Из [2, теорема 0.7] немедленно вытекает

ТЕОрема 1.1. Если многообразие $X$ является $M$-многообразием и отображение иикла

$$
\operatorname{cl}_{\mathbb{C}}: A^{<k}(X) \rightarrow H^{<2 k}(X(\mathbb{C}), \mathbb{Z} / 2)
$$

эпиморфно, то гомоморфизм

$$
\varrho_{k}: H_{\mathrm{alg}}^{k}(X(\mathbb{R}), \mathbb{Z} / 2) \rightarrow H_{\mathrm{alg}}^{2 k}(X(\mathbb{C}), \mathbb{Z} / 2)
$$

будет мономорфизмом.

В формулировке этой теоремы используются обозначения

$$
A^{<k}(X)=\bigoplus_{q<k} A^{q}(X), \quad H^{<2 k}(X(\mathbb{C}), \mathbb{Z} / 2)=\bigoplus_{q<2 k} H^{q}(X(\mathbb{C}), \mathbb{Z} / 2) .
$$

Заметим, что для полного пересечения условие теоремы вьполняется при $k<\operatorname{dim} X$.

Из результатов в работе [3] вытекает

ТЕОрема 1.2. Если многообразие $X$ является $M$-многообразием и 2-примарная подгруппа $H^{2}(X(\mathbb{C}), \mathbb{Z})(2)$ 2-периодическая, то размерность ядра гомоморфизма

$$
\varrho_{1}: H_{\mathrm{alg}}^{1}(X(\mathbb{R}), \mathbb{Z} / 2) \rightarrow H_{\mathrm{alg}}^{2}(X(\mathbb{C}), \mathbb{Z} / 2)
$$

равна иррегулярности $q(X)$.

В этой статье мы докажем следующую теорему.

ТЕОрема 1.3. Если многообразие $X$ является $M$-многообразием, то при каждом $k$ выполняется неравенство

$$
\operatorname{dim} \operatorname{ker} \varrho_{k}+\operatorname{dim} H_{\mathrm{alg}}^{<k}(X(\mathbb{R}), \mathbb{Z} / 2) \leqslant \operatorname{dim} H^{<2 k}(X(\mathbb{C}), \mathbb{Z} / 2) .
$$

Заметим, что из неравенства в этой теореме вытекает утверждение теоремы 1.1 , но это неравенство при $k=1$ дает более слабую оценку на $\operatorname{dim} k e r \varrho_{1}$, чем равенство в предыдущей теореме; мы докажем следующую теорему, в которой устранен этот недостаток.

ТЕОрема 1.4. Если многообразие $X$ является $M$-многообразием и 2-примарная подгруппа $H^{2 k}(X(\mathbb{C}), \mathbb{Z})(2)$ 2-периодическая, то при $k$ нечетном выполняется неравенство

$$
\begin{aligned}
& \operatorname{dim} \operatorname{ker} \varrho_{k}+\operatorname{dim} H_{\text {alg - odd }}^{<k}(X(\mathbb{R}), \mathbb{Z} / 2) \\
& \quad \leqslant \operatorname{dim} H^{2}\left(G, H_{\text {odd }}^{<2 k}(X(\mathbb{C}), \mathbb{Z})\right)+\operatorname{dim} H^{1}\left(G, H_{\text {even }}^{<2 k}(X(\mathbb{C}), \mathbb{Z})\right),
\end{aligned}
$$

а при $k$ четном выполняется неравенство

$$
\begin{aligned}
& \operatorname{dim} \operatorname{ker} \varrho_{k}+\operatorname{dim} H_{\text {alg }- \text { even }}^{<k}(X(\mathbb{R}), \mathbb{Z} / 2) \\
& \quad \leqslant \operatorname{dim} H^{2}\left(G, H_{\text {even }}^{<2 k}(X(\mathbb{C}), \mathbb{Z})\right)+\operatorname{dim} H^{1}\left(G, H_{\text {odd }}^{<2 k}(X(\mathbb{C}), \mathbb{Z})\right) .
\end{aligned}
$$


В этой теореме $G=G(\mathbb{C} / \mathbb{R})$ - групша Галуа, она действует на множестве комплексных точек $X(\mathbb{C})$, а поэтому и на группах когомологий. Кроме этого применяются обозначения

$$
\begin{aligned}
H_{\mathrm{alg}-\text { odd }}^{<k}(\cdot, \cdot) & =\bigoplus_{2 q-1<k} H_{\mathrm{alg}}^{2 q-1}(\cdot, \cdot), \quad H_{\mathrm{alg}-\mathrm{even}}^{<k}(\cdot, \cdot)=\bigoplus_{2 q<k} H_{\mathrm{alg}}^{2 q}(\cdot, \cdot), \\
H_{\mathrm{odd}}^{<2 k}(\cdot, \cdot)= & \bigoplus_{2 q-1<2 k} H^{2 q-1}(\cdot, \cdot), \quad H_{\mathrm{even}}^{<2 k}(\cdot, \cdot)=\bigoplus_{2 q<2 k} H^{2 q}(\cdot, \cdot),
\end{aligned}
$$

и аналогичный смысл имеют выражения

$$
H_{\text {alg - odd }}^{\leqslant k}(\cdot, \cdot), \quad H_{\text {alg }- \text { even }}^{\leqslant k}(\cdot, \cdot), \quad H_{\text {odd }}^{\leqslant 2 k}(\cdot, \cdot), \quad H_{\text {even }}^{\leqslant 2 k}(\cdot, \cdot) .
$$

Так как группы $H^{1}(G, \cdot), H^{2}(G, \cdot)$ 2-периодические, то определены их размерности над полем $\mathbb{Z} / 2$. Заметим также, что если группа $H^{q}(X(C), \mathbb{Z})$ свободная, то для $M$-многообразия вьполняются равенства

$$
\begin{aligned}
\operatorname{dim} H^{2}\left(G, H^{q}(X(C), \mathbb{Z})\right) & =\operatorname{rk} H^{q}(X(C), \mathbb{Z})_{+}, \\
\operatorname{dim} H^{1}\left(G, H^{q}(X(C), \mathbb{Z})\right) & =\operatorname{rk} H^{q}(X(C), \mathbb{Z})_{-},
\end{aligned}
$$

где $H^{q}(X(C), \mathbb{Z})_{ \pm}-$инвариантная и антиинвариантная подгрупшы группы $H^{q}(X(C), \mathbb{Z})$ относительно инволюции комплексного сопряжения $g \in G$. Если 2-примарная подгруппа $H^{q}(X(\mathbb{C}), \mathbb{Z})(2)$ 2-периодическая, то для $M$-многообразия вьполняются равенства

$\operatorname{dim} H^{2}\left(G, H^{q}(X(C), \mathbb{Z})\right)=\operatorname{dim} H^{q}(X(\mathbb{C}), \mathbb{Z})(2)+\operatorname{rk}\left(H^{q}(X(C), \mathbb{Z}) / \text { Tors }\right)_{+}$, $\operatorname{dim} H^{1}\left(G, H^{q}(X(C), \mathbb{Z})\right)=\operatorname{dim} H^{q}(X(\mathbb{C}), \mathbb{Z})(2)+\operatorname{rk}\left(H^{q}(X(C), \mathbb{Z}) / \text { Tors }\right)_{-}$.

В дополнение к сформулированным теоремам о гомоморфизме $\varrho_{*}$ будут доказаны следующие аналоги неравенства Гарнака-Тома для алгебраических когомологий. В этих теоремах многообразие $X$ не обязано быть $M$-многообразием.

Теорема 1.5. При каждом $k$ выполняется неравенство

$$
\operatorname{dim} H_{\text {alg }}^{\leqslant k}(X(\mathbb{R}), \mathbb{Z} / 2) \leqslant \operatorname{dim} H^{\leqslant 2 k}(X(\mathbb{C}), \mathbb{Z} / 2) .
$$

ТЕОрема 1.6. При каждом $k$ нечетном выполняется неравенство $\operatorname{dim} H_{\text {alg - odd }}^{\leqslant k}(X(\mathbb{R}), \mathbb{Z} / 2) \leqslant \operatorname{dim} H^{2}\left(G, H_{\text {odd }}^{<2 k}(X(\mathbb{C}), \mathbb{Z})\right)+\operatorname{dim} H^{1}\left(G, H_{\text {even }}^{\leqslant 2 k}(X(\mathbb{C}), \mathbb{Z})\right)$, а при $k$ четном -

$\operatorname{dim} H_{\text {alg - even }}^{\leqslant k}(X(\mathbb{R}), \mathbb{Z} / 2) \leqslant \operatorname{dim} H^{2}\left(G, H_{\text {even }}^{\leqslant 2 k}(X(\mathbb{C}), \mathbb{Z})\right)+\operatorname{dim} H^{1}\left(G, H_{\text {odd }}^{<2 k}(X(\mathbb{C}), \mathbb{Z})\right)$.

Заметим, что неравенство (1.1) нельзя заменить на неравенство для всех когомологий $\operatorname{dim} H^{\leqslant k}(X(\mathbb{R}), \mathbb{Z} / 2) \leqslant \operatorname{dim} H^{\leqslant 2 k}(X(\mathbb{C}), \mathbb{Z} / 2)$

при $k<n=\operatorname{dim} X$. Например, если число компонент связности $s>1$, то неравенство

$$
\operatorname{dim} H^{0}(X(\mathbb{R}), \mathbb{Z} / 2) \leqslant \operatorname{dim} H^{0}(X(\mathbb{C}), \mathbb{Z} / 2)
$$

не вьполняется. На самом деле, справедливо следующее неравенство (см. [2]):

$$
\operatorname{dim} H^{\leqslant k}(X(\mathbb{R}), \mathbb{Z} / 2) \leqslant \operatorname{dim} H^{\leqslant k+n}(X(\mathbb{C}), \mathbb{Z} / 2) .
$$


2. Применение эквивариантных когомологий с коэффициентами в $\mathbb{Z} / 2$. При доказательстве теорем 1.3, 1.5 мы будем применять эквивариантные когомологии $H^{*}(X(\mathbb{C}) ; G, \mathbb{Z} / 2)$ и эквивариантное отображение цикла. Сначала мы приведем без доказательства необходимые далее факты об эквивариантных когомологиях вещественного алгебраического многообразия. Подробности можно посмотреть в [1]-[8].

На множестве комплексных точек $X(\mathbb{C})$ действует группа Галуа $G=G(\mathbb{C} / \mathbb{R})$, поэтому определены эквивариантные когомологии $H^{*}(X(\mathbb{C}) ; G, \mathbb{Z} / 2)$. Имеются две спектральные последовательности, которые сходятся к этим когомологиям. Первая спектральная последовательность нам не потребуется, поэтому мы ее не приводим, а вторая спектральная последовательность имеет вид

$$
I I_{2}^{p, q}=H^{p}\left(G, H^{q}(X(\mathbb{C}), \mathbb{Z} / 2)\right) \Rightarrow H^{p+q}(X(\mathbb{C}) ; G, \mathbb{Z} / 2) .
$$

Для $M$-многообразия эта спектральная последовательность вырождается, причем

$$
I I_{2}^{p, q}=H^{p}\left(G, H^{q}(X(\mathbb{C}), \mathbb{Z} / 2)\right)=H^{q}(X(\mathbb{C}), \mathbb{Z} / 2)
$$

Обозначим через $t$ образующую групшы

$$
H^{1}(\mathrm{pt} ; G, \mathbb{Z} / 2)=H^{1}(G, \mathbb{Z} / 2)
$$

где $\mathrm{pt}$ - одноточечное $G$-пространство. Тогда гомоморфизм умножения на $t$ будем обозначать через

$$
t: H^{q}(X(\mathbb{C}) ; G, \mathbb{Z} / 2) \rightarrow H^{q+1}(X(\mathbb{C}) ; G, \mathbb{Z} / 2)
$$

и назьвать гомоморфизмом сдвига (см. [7]), для $M$-многообразия он является вложением. Спектральная последовательность (2.1) определяет возрастающую фильтрацию

$$
\begin{aligned}
F^{0} H^{q}(X(\mathbb{C}) ; G, \mathbb{Z} / 2) & \subset F^{1} H^{q}(X(\mathbb{C}) ; G, \mathbb{Z} / 2) \subset \cdots \subset F^{q-1} H^{q}(X(\mathbb{C}) ; G, \mathbb{Z} / 2) \\
& \subset F^{q} H^{q}(X(\mathbb{C}) ; G, \mathbb{Z} / 2)=H^{q}(X(\mathbb{C}) ; G, \mathbb{Z} / 2)
\end{aligned}
$$

причем выполняются равенства

$$
\begin{aligned}
F^{p} H^{q}(X(\mathbb{C}) ; G, \mathbb{Z} / 2) & =t^{q-p} H^{p}(X(\mathbb{C}) ; G, \mathbb{Z} / 2), \\
F^{q-1} H^{q}(X(\mathbb{C}) ; G, \mathbb{Z} / 2) & =\operatorname{ker}\left[\alpha: H^{q}(X(\mathbb{C}) ; G, \mathbb{Z} / 2) \rightarrow H^{q}(X(\mathbb{C}), \mathbb{Z} / 2)\right],
\end{aligned}
$$

где $\alpha$ - канонический гомоморфизм забьвания вещественной структуры. Мы можем рассмотреть эквивариантные когомологии множества вещественных точек, тогда выполняется равенство

$$
H^{q}(X(\mathbb{R}) ; G, \mathbb{Z} / 2)=\bigoplus_{k=0}^{q} H^{k}(X(\mathbb{R}), \mathbb{Z} / 2)
$$

Заметим, что при гомоморфизме ограничения

$$
\beta: H^{q}(X(\mathbb{C}) ; G, \mathbb{Z} / 2) \rightarrow H^{q}(X(\mathbb{R}) ; G, \mathbb{Z} / 2)=\bigoplus_{k=0}^{q} H^{k}(X(\mathbb{R}), \mathbb{Z} / 2)
$$


образы элементов

$$
a \in H^{p}(X(\mathbb{R}) ; G, \mathbb{Z} / 2), \quad t^{q-p} a \in H^{q}(X(\mathbb{R}) ; G, \mathbb{Z} / 2)
$$

совпадают, т.е. вьполняется равенство $\beta\left(t^{q-p} a\right)=\beta(a)$. Рассмотрим возрастающую фильтрацию

$$
\mathscr{F}^{0} \subset \mathscr{F}^{0} \subset \cdots \subset \mathscr{F}^{2 n-1} \subset \mathscr{F}^{2 n}=H^{*}(X(\mathbb{R}), \mathbb{Z} / 2),
$$

где

$$
\mathscr{F}^{p}=\beta\left(H^{p}(X(\mathbb{C}) ; G, \mathbb{Z} / 2)\right) \subset H^{\leqslant p}(X(\mathbb{R}), \mathbb{Z} / 2) \subset H^{*}(X(\mathbb{R}), \mathbb{Z} / 2) .
$$

Эта фильтрация получается из спектральной последовательности, которую мы сейчас опишем. При $p+q>2 n$ группа $I I_{r}^{p, q}$ не зависит от $p$; обозначим ее через $H_{r}^{q}$. Дифференциал

$$
d_{r}^{p, q}: I I_{r}^{p, q} \rightarrow I I_{r}^{p+r, q-r+1}
$$

также не зависит от $p$ при $p+q>2 n$; обозначим его через $d_{r}^{q}$. Тогда получаем спектральную последовательность $\left(H_{r}^{q}, d_{r}^{q}\right)$, которая сходится к групше $H^{N}(X(\mathbb{C}) ; G, \mathbb{Z} / 2)$, где $N>2 n$. Гомоморфизм ограничения

$$
\beta: H^{N}(X(\mathbb{C}) ; G, \mathbb{Z} / 2) \rightarrow H^{N}(X(\mathbb{R}) ; G, \mathbb{Z} / 2)=H^{*}(X(\mathbb{R}), \mathbb{Z} / 2)
$$

является изоморфизмом при $N>2 n$, поэтому можно считать, что спектральная последовательность $\left(H_{r}^{q}, d_{r}^{q}\right)$ сходится к групе $H^{*}(X(\mathbb{R}), \mathbb{Z} / 2)$. Эта спектральная последовательность в работе [4] названа спектральной последовательностью Калинина, так как Калинин заметил в [5], что удобно вместо всей второй спектральной последовательности $I I_{r}^{p, q}$ рассматривать полученную предельную спектральную последовательность $H_{r}^{q}$. Заметим, что

$$
H_{2}^{q}=H^{1}\left(G, H^{q}(X(\mathbb{C}), \mathbb{Z} / 2)\right)=H^{q}(X(\mathbb{C}), \mathbb{Z} / 2)^{G} /\left(1+g^{*}\right) H^{q}(X(\mathbb{C}), \mathbb{Z} / 2) .
$$

Фильтрация (2.2) получается из этой спектральной последовательности.

Кроме комплексного (0.2) и вещественного (0.1) отображений цикла определено эквивариантное отображение цикла

$$
\mathrm{cl}: A^{*}(X) \rightarrow H^{*}(X(\mathbb{C}) ; G, \mathbb{Z} / 2)
$$

которое циклу $Z \in A^{q}(X)$ сопоставляет эквивариантньй класс когомологий

$$
[Z(\mathbb{C}) ; G]^{*} \in H^{2 q}(X(\mathbb{C}) ; G, \mathbb{Z} / 2)
$$

Эти отображения цикла связаны между собой соотношениями

$$
\alpha \circ \mathrm{cl}=\mathrm{cl}_{\mathbb{C}}, \quad \beta \circ \mathrm{cl}=S q \circ \mathrm{cl}_{\mathbb{R}}
$$

где

$$
\beta: H^{2 q}(X(\mathbb{C}) ; G, \mathbb{Z} / 2) \rightarrow H^{2 q}(X(\mathbb{R}) ; G, \mathbb{Z} / 2)=\bigoplus_{k=0}^{2 q} H^{k}(X(\mathbb{R}), \mathbb{Z} / 2)
$$

- гомоморфизм ограничения, а $S q$ - полный квадрат Стинрода (см. [1], [2]). 
ЛЕмма 2.1. Для каждого $k$ выполняется включение

$$
S q\left(H_{\mathrm{alg}}^{k}(X(\mathbb{R}), \mathbb{Z} / 2)\right) \subset \mathscr{F}^{2 k} .
$$

ДокАЗАтЕЛЬСТво. Пусть $a \in H_{\mathrm{alg}}^{k}(X(\mathbb{R}), \mathbb{Z} / 2)$ и $Z \in A^{k}(X)$ - такой цикл, что $\operatorname{cl}_{\mathbb{R}}(Z)=a$; тогда $\operatorname{cl}(Z) \in H^{2 k}(X(\mathbb{C}) ; G, \mathbb{Z} / 2)$, поэтому $\beta(\operatorname{cl}(Z)) \in \mathscr{F}^{2 k}$. С другой стороны, имеем равенства $\beta(\operatorname{cl}(Z))=S q\left(\operatorname{cl}_{\mathbb{R}}(Z)\right)=S q(a)$. Лемма доказана.

Из этой леммы вытекает включение

$$
S q\left(H_{\mathrm{alg}}^{\leqslant k}(X(\mathbb{R}), \mathbb{Z} / 2)\right) \subset \mathscr{F}^{2 k} .
$$

Так как гомоморфизм

$$
S q: H^{*}(X(\mathbb{R}), \mathbb{Z} / 2) \rightarrow H^{*}(X(\mathbb{R}), \mathbb{Z} / 2)
$$

является изоморфизмом, то справедливы неравенства

$$
\begin{aligned}
\operatorname{dim} H_{\mathrm{alg}}^{\leqslant k}(X(\mathbb{R}), \mathbb{Z} / 2) & =\operatorname{dim} S q\left(H_{\mathrm{alg}}^{\leqslant k}(X(\mathbb{R}), \mathbb{Z} / 2)\right) \leqslant \operatorname{dim} \mathscr{F}^{2 k}=\operatorname{dim} H_{\infty}^{\leqslant 2 k} \\
& \leqslant H_{2}^{\leqslant 2 k}=\operatorname{dim} H^{1}\left(G, H^{\leqslant 2 k}(X(\mathbb{C}), \mathbb{Z} / 2)\right) \leqslant H^{\leqslant 2 k}(X(\mathbb{C}), \mathbb{Z} / 2) .
\end{aligned}
$$

Итак, теорема 1.5 доказана, переходим к доказательству теоремы 1.3. Рассмотрим группу

$$
S q\left(\operatorname{ker} \varrho_{k} \oplus H_{\text {alg }}^{<k}(X(\mathbb{R}), \mathbb{Z} / 2)\right) .
$$

Покажем, что она содержится в групше $\mathscr{F}^{2 k-1}$. В силу леммы 2.1 группа

$$
S q\left(H_{\mathrm{alg}}^{<k}(X(\mathbb{R}), \mathbb{Z} / 2)\right)
$$

содержится в группе $\mathscr{F}^{2 k-2}$, а поэтому она содержится в группе $\mathscr{F}^{2 k-1}$. Пусть $a \in \operatorname{ker} \varrho_{k}$ и $Z \in A^{k}(X)$ - такой цикл, что $\operatorname{cl}_{\mathbb{R}}(Z)=a$; тогда элемент

$$
\operatorname{cl}_{\mathbb{C}}(Z)=\alpha(\operatorname{cl}(Z)) \in H^{2 k}(X(\mathbb{C}), \mathbb{Z} / 2)
$$

равен нулю. Следовательно,

$$
\operatorname{cl}(Z) \in F^{2 k-1} H^{2 k}(X(\mathbb{C}) ; G, \mathbb{Z} / 2)=t H^{2 k-1}(X(\mathbb{C}) ; G, \mathbb{Z} / 2) .
$$

Поэтому

$$
S q(a)=\beta(\operatorname{cl}(Z)) \in \beta\left(H^{2 k-1}(X(\mathbb{C}) ; G, \mathbb{Z} / 2)\right)=\mathscr{F}^{2 k-1} .
$$

Итак, включение

$$
S q\left(\operatorname{ker} \varrho_{k} \oplus H_{\mathrm{alg}}^{<k}(X(\mathbb{R}), \mathbb{Z} / 2)\right) \subset \mathscr{F}^{2 k-1}
$$

доказано, поэтому выполняются неравенства

$$
\begin{aligned}
\operatorname{dim} \operatorname{ker} \varrho_{k}+\operatorname{dim} H_{\text {alg }}^{<k}(X(\mathbb{R}), \mathbb{Z} / 2) & =\operatorname{dim} S q\left(\operatorname{ker} \varrho_{k} \oplus H_{\text {alg }}^{<k}(X(\mathbb{R}), \mathbb{Z} / 2)\right) \\
& \leqslant \operatorname{dim} \mathscr{F}^{2 k-1}=\operatorname{dim} H_{\infty}^{<2 k} \leqslant H_{2}^{<2 k} \\
& =\operatorname{dim} H^{1}\left(G, H^{<2 k}(X(\mathbb{C}), \mathbb{Z} / 2)\right) \\
& \leqslant H^{<2 k}(X(\mathbb{C}), \mathbb{Z} / 2) .
\end{aligned}
$$

Теорема 1.3 доказана. 


\section{3. Применение эквивариантных когомологий с целыми коэффициента-} ми. При доказательстве теорем $1.4,1.6$ мы будем применять эквивариантные когомологии с целыми коэффициентами $H^{*}(X(\mathbb{C}) ; G, \mathbb{Z})$. Имеются две спектральные последовательности, которые сходятся к этим когомологиям. Первая спектральная последовательность нам не потребуется, поэтому мы ее не приводим, а вторая спектральная последовательность имеет вид

$$
I I_{2}^{p, q}(\mathbb{Z})=H^{p}\left(G, H^{q}(X(\mathbb{C}), \mathbb{Z})\right) \Rightarrow H^{p+q}(X(\mathbb{C}) ; G, \mathbb{Z}) .
$$

Пусть $\mathbb{Z}{ }_{-}-G$-модуль целых чисел, на котором инволюция $g \in G$ действует умножением на -1. Тогда мы можем рассмотреть эквивариантные когомологии $H^{*}\left(X(\mathbb{C}) ; G, \mathbb{Z} \_\right)$. Вторая спектральная последовательность для них имеет вид

$$
I I_{2}^{p, q}\left(\mathbb{Z}_{-}\right)=H^{p}\left(G, H^{q}\left(X(\mathbb{C}), \mathbb{Z}_{-}\right)\right) \Rightarrow H^{p+q}\left(X(\mathbb{C}) ; G, \mathbb{Z}_{-}\right) .
$$

$G$-модуль целых чисел, на котором инволюция $g \in G$ действует тривиально, мы будем обозначать через $\mathbb{Z}_{+}$или просто через $\mathbb{Z}$. Итак,

$$
H^{*}\left(X(\mathbb{C}) ; G, \mathbb{Z}_{+}\right)=H^{*}(X(\mathbb{C}) ; G, \mathbb{Z})
$$

Обозначим через $T$ образующую группы

$$
H^{1}\left(\mathrm{pt} ; G, \mathbb{Z}_{-}\right)=H^{1}\left(G, \mathbb{Z}_{-}\right)=\mathbb{Z} / 2,
$$

где $\mathrm{pt}$ - одноточечное $G$-пространство. Тогда гомоморфизм умножения на $T$ будем обозначать через

$$
T: H^{q}\left(X(\mathbb{C}) ; G, \mathbb{Z}_{ \pm}\right) \rightarrow H^{q+1}\left(X(\mathbb{C}) ; G, \mathbb{Z}_{\mp}\right)
$$

и назьвать гомоморфизмом сдвига (см. [3], [7]). Спектральная последовательность (3.1) определяет возрастающую фильтрацию

$$
\begin{aligned}
F^{0} H^{q}(X(\mathbb{C}) ; G, \mathbb{Z}) & \subset F^{1} H^{q}(X(\mathbb{C}) ; G, \mathbb{Z}) \subset \cdots \subset F^{q-1} H^{q}(X(\mathbb{C}) ; G, \mathbb{Z}) \\
& \subset F^{q} H^{q}(X(\mathbb{C}) ; G, \mathbb{Z})=H^{q}(X(\mathbb{C}) ; G, \mathbb{Z}),
\end{aligned}
$$

аналогичную фильтрацию групшы $H^{q}(X(\mathbb{C}) ; G, \mathbb{Z}-)$ определяет спектральная последовательность (3.2). Заметим, что $G$-модуль $\mathbb{Z}$ - обозначается также через $\mathbb{Z}(1)$, а вообще, через $\mathbb{Z}(l)$ обозначается $G$-модуль целых чисел, на котором инволюция $g \in G$ действует умножением на $(-1)^{l}$. Таким образом, при четном $l$ имеем равенство $\mathbb{Z}(l)=\mathbb{Z}$, а при нечетном $l$ справедливо равенство $\mathbb{Z}(l)=\mathbb{Z}$. . Тогда при $p \leqslant q$ выполняется равенство

$$
F^{p} H^{q}(X(\mathbb{C}) ; G, \mathbb{Z}(l))=T^{q-p} H^{p}(X(\mathbb{C}) ; G, \mathbb{Z}(l+p-q)) .
$$

Мы можем рассмотреть эквивариантные когомологии множества вещественных точек. Заметим, что при $l$ четном вьполняется равенство

$$
H^{q}(X(\mathbb{R}) ; G, \mathbb{Z}(l))=H^{q}(X(\mathbb{R}), \mathbb{Z}) \oplus H^{q-2}(X(\mathbb{R}), \mathbb{Z} / 2) \oplus H^{q-4}(X(\mathbb{R}), \mathbb{Z} / 2) \oplus \cdots
$$

и гомоморфизм редукции по $\bmod 2$

$$
R: H^{q}(X(\mathbb{R}) ; G, \mathbb{Z}(l)) \rightarrow H^{q}(X(\mathbb{R}) ; G, \mathbb{Z} / 2)=\bigoplus_{p=0}^{q} H^{p}(X(\mathbb{R}), \mathbb{Z} / 2)
$$


переводит сумму $\left[a^{q}\right]+\left[a^{q-2}\right]+\left[a^{q-4}\right]+\cdots$ в сумму

$$
\left[a^{q} \bmod 2\right]+\delta\left(\left[a^{q-2}\right]\right)+\left[a^{q-2}\right]+\delta\left(\left[a^{q-4}\right]\right)+\left[a^{q-4}\right]+\cdots,
$$

где $\delta$ - гомоморфизм Бокштейна. А при $l$ нечетном выполняется равенство

$$
H^{q}(X(\mathbb{R}) ; G, \mathbb{Z}(l))=H^{q-1}(X(\mathbb{R}), \mathbb{Z} / 2) \oplus H^{q-3}(X(\mathbb{R}), \mathbb{Z} / 2) \oplus H^{q-5}(X(\mathbb{R}), \mathbb{Z} / 2) \oplus \cdots
$$

и гомоморфизм редукции по $\bmod 2$ переводит сумму $\left[a^{q-1}\right]+\left[a^{q-3}\right]+\left[a^{q-5}\right]+\cdots$ в сумму

$$
\delta\left(\left[a^{q-1}\right]\right)+\left[a^{q-1}\right]+\delta\left(\left[a^{q-3}\right]\right)+\left[a^{q-3}\right]+\delta\left(\left[a^{q-5}\right]\right)+\left[a^{q-5}\right]+\cdots .
$$

\section{Обозначим через}

$B: H^{q}(X(\mathbb{C}) ; G, \mathbb{Z}(l)) \rightarrow H^{q-1}(X(\mathbb{R}), \mathbb{Z} / 2) \oplus H^{q-3}(X(\mathbb{R}), \mathbb{Z} / 2) \oplus H^{q-5}(X(\mathbb{R}), \mathbb{Z} / 2) \oplus \cdots$ гомоморфизм ограничения

$$
H^{q}(X(\mathbb{C}) ; G, \mathbb{Z}(l)) \rightarrow H^{q}(X(\mathbb{R}) ; G, \mathbb{Z}(l))
$$

при нечетном $l$, и пусть при четном $l$ гомоморфизм

$B: H^{q}(X(\mathbb{C}) ; G, \mathbb{Z}(l)) \rightarrow H^{q}(X(\mathbb{R}), \mathbb{Z} / 2) \oplus H^{q-2}(X(\mathbb{R}), \mathbb{Z} / 2) \oplus H^{q-4}(X(\mathbb{R}), \mathbb{Z} / 2) \oplus \cdots$ равен композиции гомоморфизма ограничения и гомоморфизма редукции на первом слагаемом

$$
\begin{aligned}
& H^{q}(X(\mathbb{R}), \mathbb{Z}) \oplus H^{q-2}(X(\mathbb{R}), \mathbb{Z} / 2) \oplus H^{q-4}(X(\mathbb{R}), \mathbb{Z} / 2) \oplus \cdots \\
& \quad \rightarrow H^{q}(X(\mathbb{R}), \mathbb{Z} / 2) \oplus H^{q-2}(X(\mathbb{R}), \mathbb{Z} / 2) \oplus H^{q-4}(X(\mathbb{R}), \mathbb{Z} / 2) \oplus \cdots .
\end{aligned}
$$

Через $H_{\text {odd }}^{*}(X(\mathbb{R}), \mathbb{Z} / 2)$ обозначим прямую сумму

$$
\bigoplus_{q} H^{2 q-1}(X(\mathbb{R}), \mathbb{Z} / 2)
$$

аналогично,

$$
H_{\text {even }}^{*}(X(\mathbb{R}), \mathbb{Z} / 2)=\bigoplus_{q} H^{2 q}(X(\mathbb{R}), \mathbb{Z} / 2) .
$$

На этих группах определены возрастающие фильтрации

$$
\begin{gathered}
\mathscr{F}_{\text {odd }}^{0} \subset \mathscr{F}_{\text {odd }}^{1} \subset \cdots \subset \mathscr{F}_{\text {odd }}^{2 n-1} \subset \mathscr{F}_{\text {odd }}^{2 n}=H_{\text {odd }}^{*}(X(\mathbb{R}), \mathbb{Z} / 2), \\
\mathscr{F}_{\text {even }}^{0} \subset \mathscr{F}_{\text {even }}^{1} \subset \cdots \subset \mathscr{F}_{\text {even }}^{2 n-1} \subset \mathscr{F}_{\text {even }}^{2 n}=H_{\text {even }}^{*}(X(\mathbb{R}), \mathbb{Z} / 2),
\end{gathered}
$$

где

$$
\begin{aligned}
& \mathscr{F}_{\text {odd }}^{p}=B\left(H^{p}(X(\mathbb{C}) ; G, \mathbb{Z}(p+1))\right) \subset H_{\text {odd }}^{*}(X(\mathbb{R}), \mathbb{Z} / 2), \\
& \mathscr{F}_{\text {even }}^{p}=B\left(H^{p}(X(\mathbb{C}) ; G, \mathbb{Z}(p))\right) \subset H_{\text {even }}^{*}(X(\mathbb{R}), \mathbb{Z} / 2) .
\end{aligned}
$$

Эти фильтрации получаются из спектральных последовательностей Калинина

$$
\left(H_{r}^{\prime q}, d_{r}^{\prime q}\right) \Rightarrow H_{\text {odd }}^{*}(X(\mathbb{R}), \mathbb{Z} / 2), \quad\left(H_{r}^{\prime \prime}{ }_{r}, d^{\prime \prime}{ }_{r}^{q}\right) \Rightarrow H_{\text {even }}^{*}(X(\mathbb{R}), \mathbb{Z} / 2),
$$

которые строятся так же, как спектральная последовательность $\left(H_{r}^{q}, d_{r}^{q}\right)$. Заметим, что выполняются равенства

$$
\begin{aligned}
H_{2}^{\prime 2 q-1} & =H^{2}\left(G, H^{2 q-1}(X(\mathbb{C}), \mathbb{Z})\right), & H_{2}^{\prime 2 q} & =H^{1}\left(G, H^{2 q}(X(\mathbb{C}), \mathbb{Z})\right), \\
H_{2}^{\prime \prime 2 q-1} & =H^{1}\left(G, H^{2 q-1}(X(\mathbb{C}), \mathbb{Z}),\right. & H_{2}^{\prime 2 q} & =H^{2}\left(G, H^{2 q}(X(\mathbb{C}), \mathbb{Z}) .\right.
\end{aligned}
$$

Определены гомоморфизмы редукции по $\bmod 2$

$$
\left(H_{r}^{\prime q}, d_{r}^{\prime q}\right) \rightarrow\left(H_{r}^{q}, d_{r}^{q}\right), \quad\left(H_{r}^{\prime q}, d_{r}^{\prime q}\right) \rightarrow\left(H_{r}^{q}, d_{r}^{q}\right)
$$

заметим, что при этом соответствующие гомоморфизмы

$$
H_{\text {odd }}^{*}(X(\mathbb{R}), \mathbb{Z} / 2) \rightarrow H^{*}(X(\mathbb{R}), \mathbb{Z} / 2), \quad H_{\text {even }}^{*}(X(\mathbb{R}), \mathbb{Z} / 2) \rightarrow H^{*}(X(\mathbb{R}), \mathbb{Z} / 2)
$$

равны $1+\delta$, где $\delta$ - гомоморфизм Бокштейна. 
ЛЕмма 3.1. При каждом нечетном $k$ выполняется включение

$$
S q^{\text {even }}\left(H_{\text {alg }}^{k}(X(\mathbb{R}), \mathbb{Z} / 2)\right) \subset \mathscr{F}_{\text {odd }}^{2 k},
$$

а при четном $k$ -

$$
S q^{\text {even }}\left(H_{\text {alg }}^{k}(X(\mathbb{R}), \mathbb{Z} / 2)\right) \subset \mathscr{F}_{\text {even }}^{2 k},
$$

əде $S q^{\text {even }}=1+S q^{2}+S q^{4}+\cdots$.

ДокАЗАТЕльСтво. Мы рассмотрели отображения цикла $(0.2),(2.3)$ в когомологии с коэффициентами в $\mathbb{Z} / 2$, но определены также отображения цикла в когомологии с целыми коэффициентами

$$
\mathrm{CL}_{\mathbb{C}}: A^{k}(X) \rightarrow H^{2 k}(X(\mathbb{C}), \mathbb{Z}), \quad \mathrm{CL}: A^{k}(X) \rightarrow H^{2 k}(X(\mathbb{C}) ; G, \mathbb{Z}(k)),
$$

композиция которых с гомоморфизмом редукции по mod 2 совпадает с гомоморфизмами $(0.2),(2.3)$. Из равенства $\beta$ ० $\mathrm{cl}=S q$ ० $\mathrm{cl}_{\mathbb{R}}$ вытекает равенство $B \circ \mathrm{CL}=S q^{\text {even }} \circ \mathrm{cl}_{\mathbb{R}}$. Из этого соотношения и вытекают утверждения леммы. Лемма доказана.

Из этой леммы вытекают включения

$$
S q^{\text {even }}\left(H_{\text {alg - odd }}^{\leqslant k}(X(\mathbb{R}), \mathbb{Z} / 2)\right) \subset \mathscr{F}_{\text {odd }}^{2 k}, \quad S q^{\text {even }}\left(H_{\text {alg - even }}^{\leqslant k}(X(\mathbb{R}), \mathbb{Z} / 2)\right) \subset \mathscr{F}_{\text {even }}^{2 k} .
$$

Так как выполняется равенство $S q=(1+\delta) \circ S q^{\text {even }}$, то гомоморфизм

$$
S q^{\text {even }}: H^{*}(X(\mathbb{R}), \mathbb{Z} / 2) \rightarrow H^{*}(X(\mathbb{R}), \mathbb{Z} / 2)
$$

является изоморфизмом. Поэтому справедливы неравенства: если $k$ нечетное, то

$$
\begin{aligned}
& \operatorname{dim} H_{\text {alg- odd }}^{\leqslant k}(X(\mathbb{R}), \mathbb{Z} / 2)=\operatorname{dim} S q^{\text {even }}\left(H_{\text {alg }- \text { odd }}^{\leqslant k}(X(\mathbb{R}), \mathbb{Z} / 2)\right) \\
& \quad \leqslant \operatorname{dim} \mathscr{F}_{\text {odd }}^{2 k}=\operatorname{dim} H_{\infty}^{\prime \leqslant 2 k} \leqslant H_{2}^{\prime \leqslant 2 k} \\
& =\operatorname{dim} H^{2}\left(G, H_{\text {odd }}^{<2 k}(X(\mathbb{C}), \mathbb{Z})\right)+\operatorname{dim} H^{1}\left(G, H_{\text {even }}^{\leqslant 2 k}(X(\mathbb{C}), \mathbb{Z})\right),
\end{aligned}
$$

а если $k$ четное, то

$$
\begin{aligned}
\operatorname{dim} & H_{\text {alg }- \text { even }}^{\leqslant k}(X(\mathbb{R}), \mathbb{Z} / 2)=\operatorname{dim} S q^{\text {even }}\left(H_{\text {alg }- \text { even }}^{\leqslant k}(X(\mathbb{R}), \mathbb{Z} / 2)\right) \\
& \leqslant \operatorname{dim} \mathscr{F}_{\text {even }}^{2 k}=\operatorname{dim} H_{\infty}^{\prime \prime \leqslant 2 k} \leqslant H_{2}^{\prime \prime} \leqslant 2 k \\
& =\operatorname{dim} H^{2}\left(G, H_{\text {even }}^{\leqslant 2 k}(X(\mathbb{C}), \mathbb{Z})\right)+\operatorname{dim} H^{1}\left(G, H_{\text {odd }}^{<2 k}(X(\mathbb{C}), \mathbb{Z})\right) .
\end{aligned}
$$

Итак, теорема 1.6 доказана, переходим к доказательству теоремы 1.4. Прежде всего будет доказана следующая

ЛЕмма 3.2. Пусть многообразие $X$ является $M$-многообразием, а 2-примарная подгруппа $H^{q}(X(\mathbb{C}), \mathbb{Z})(2)$ группь когомологий $H^{q}(X(\mathbb{C}), \mathbb{Z})$ является 2-периодической. Тогда при $p=1,2$ гомоморфизмы

$$
H^{p}\left(G, H^{q}(X(\mathbb{C}), \mathbb{Z})\right) \rightarrow H^{p}\left(G, H^{q}(X(\mathbb{C}), \mathbb{Z} / 2)\right),
$$

индуцированнье редукиией по $\bmod 2$

$$
R: H^{q}(X(\mathbb{C}), \mathbb{Z}) \rightarrow H^{q}(X(\mathbb{C}), \mathbb{Z} / 2),
$$

мономорфны. 
ДоКАЗАТЕЛЬСТво. Гомоморфизмы (3.4) разлагаются в композицию гомоморфизмOB

$$
H^{p}\left(G, H^{q}(X(\mathbb{C}), \mathbb{Z})\right) \rightarrow H^{p}\left(G, H^{q}(X(\mathbb{C}), \mathbb{Z}) \otimes \mathbb{Z} / 2\right) \rightarrow H^{p}\left(G, H^{q}(X(\mathbb{C}), \mathbb{Z} / 2)\right) .
$$

Так как для $M$-многообразия инволюция $g \in G$ действует на $\left.H^{q}(X(\mathbb{C}), \mathbb{Z} / 2)\right)$ тривиально, то вьполняются равенства

$$
\begin{aligned}
H^{p}\left(G, H^{q}(X(\mathbb{C}), \mathbb{Z} / 2)\right) & =H^{q}(X(\mathbb{C}), \mathbb{Z} / 2), \\
\left.H^{p}\left(G, H^{q}(X(\mathbb{C}), \mathbb{Z}) \otimes \mathbb{Z} / 2\right)\right) & \left.=H^{q}(X(\mathbb{C}), \mathbb{Z}) \otimes \mathbb{Z} / 2\right),
\end{aligned}
$$

а поэтому гомоморфизм

$$
H^{p}\left(G, H^{q}(X(\mathbb{C}), \mathbb{Z}) \otimes \mathbb{Z} / 2\right) \rightarrow H^{p}\left(G, H^{q}(X(\mathbb{C}), \mathbb{Z} / 2)\right)
$$

мономорфен. Следовательно, достаточно проверить мономорфность гомоморфизма

$$
H^{p}\left(G, H^{q}(X(\mathbb{C}), \mathbb{Z})\right) \rightarrow H^{p}\left(G, H^{q}(X(\mathbb{C}), \mathbb{Z}) \otimes \mathbb{Z} / 2\right) .
$$

Далее, для сокращения записи введем обозначения

$$
H^{q}(X(\mathbb{C}), \mathbb{Z})=H, \quad T=\text { Tors } H, \quad L=H / T
$$

и рассмотрим коммутативную диаграмму из $G$-модулей

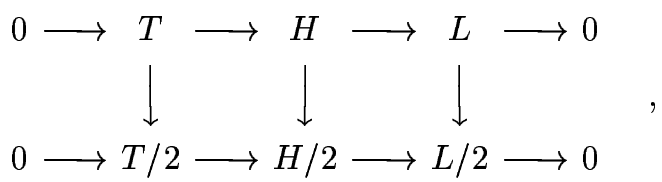

строки в которой точны. Из нее получаем коммутативную диаграмму когомологий этих $G$-модулей

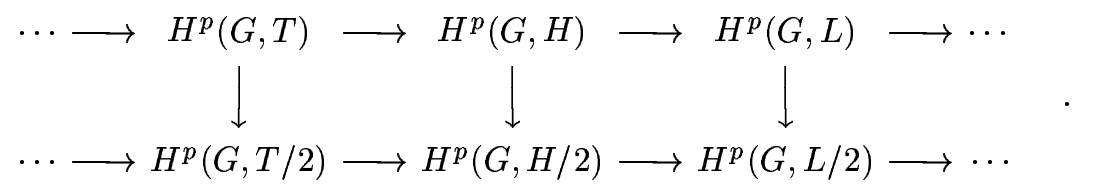

Из условия, что 2-примарная группа $T(2)$ является 2-периодической следует, что гомоморфизм

$$
H^{p}(G, T) \rightarrow H^{p}(G, T / 2)
$$

является изоморфизмом, а так как инволюция $g \in G$ действует на $H / 2$ тривиально, то гомоморфизм

$$
H^{p}(G, T / 2) \rightarrow H^{p}(G, H / 2)
$$

является мономорфизмом. Группа $L$ свободная, поэтому гомоморфизм

$$
H^{p}(G, L) \rightarrow H^{p}(G, L / 2)
$$


также мономорфен. Тогда из верхней коммутативной диаграммы следует, что гомоморфизм

$$
H^{p}(G, H) \rightarrow H^{p}(G, H / 2)
$$

мономорфен. Лемма доказана.

Мы будем доказьвать только первое неравенство в теореме 1.4 , т.е. для нечетного $k$, так как второе неравенство доказьвается аналогично. Прежде всего, покажем, что выполняется включение

$$
S q^{\text {even }}\left(\operatorname{ker} \varrho_{k} \oplus H_{\text {alg }- \text { odd }}^{<k}(X(\mathbb{R}), \mathbb{Z} / 2)\right) \subset \mathscr{F}_{\text {odd }}^{2 k-1} .
$$

В силу леммы 3.1 группа

$$
S q^{\text {even }}\left(H_{\text {alg }- \text { odd }}^{<k}(X(\mathbb{R}), \mathbb{Z} / 2)\right)
$$

содержится в группе $\mathscr{F}_{\text {odd }}^{2 k-2}$, а поэтому она содержится в группе $\mathscr{F}_{\text {odd }}^{2 k-1}$; осталось доказать включение

$$
S q^{\text {even }}\left(\operatorname{ker} \varrho_{k}\right) \subset \mathscr{F}_{\text {odd }}^{2 k-1} \text {. }
$$

Пусть $a \in \operatorname{ker} \varrho_{k}$ и $Z \in A^{k}(X)$ - такой цикл, что $\operatorname{cl}_{\mathbb{R}}(Z)=a$; тогда элемент

$$
\varrho_{k}(a)=\operatorname{cl}_{\mathbb{C}}(Z)=\alpha(\operatorname{cl}(Z)) \in H^{2 k}(X(\mathbb{C}), \mathbb{Z} / 2)
$$

равен нулю, а нужно показать, что

$$
S q^{\text {even }}(a) \in \mathscr{F}_{\text {odd }}^{2 k-1} .
$$

Рассмотрим элемент $\mathrm{CL}_{\mathbb{C}}(Z) \in H^{2 k}(X(\mathbb{C}), \mathbb{Z})$; он принадлежит группе $H^{2 k}(X(\mathbb{C}), \mathbb{Z})_{\text {_ }}$ и определяет элемент грушы

$$
{H_{2}^{\prime 2 k}}_{2}^{2 k} H^{1}\left(G, H^{2 k}(X(\mathbb{C}), \mathbb{Z})\right)=H^{2 k}(X(\mathbb{C}), \mathbb{Z})_{-} /\left(1-g^{*}\right) H^{2 k}(X(\mathbb{C}), \mathbb{Z}) .
$$

Обозначим этот элемент через $b$; тогда при гомоморфизме

$$
H^{1}\left(G, H^{2 k}(X(\mathbb{C}), \mathbb{Z})\right) \rightarrow H^{1}\left(G, H^{2 k}(X(\mathbb{C}), \mathbb{Z} / 2)\right)=H^{2 k}(X(\mathbb{C}), \mathbb{Z} / 2)
$$

он переходит в элемент $\operatorname{cl}_{\mathbb{C}}(a)$, т.е. в нуль. А из леммы 3.2 следует, что и элемент $b$ равен нулю. Поэтому класс когомологий $\mathrm{CL}_{\mathbb{C}}(Z)=\alpha(\mathrm{CL}(Z))$ определяет нулевой элемент из группы $H_{\infty}^{\prime 2 k}$. Это означает, что

$$
S q^{\text {even }}(a)=B(\mathrm{CL}(Z)) \in \mathscr{F}_{\text {odd }}^{2 k-1} .
$$

Итак, включение (3.6) доказано, поэтому выполняется включение (3.5), следовательно, справедливы неравенства

$$
\begin{aligned}
& \operatorname{dim} \operatorname{ker} \varrho_{k}+\operatorname{dim} H_{\text {alg }- \text { odd }}^{<k}(X(\mathbb{R}), \mathbb{Z} / 2)=\operatorname{dim} S q^{\text {even }}\left(\operatorname{ker} \varrho_{k} \oplus H_{\text {alg }- \text { odd }}^{<k}(X(\mathbb{R}), \mathbb{Z} / 2)\right) \\
& \leqslant \operatorname{dim} \mathscr{F}_{\text {odd }}^{2 k-1}=\operatorname{dim} H_{\infty}^{\prime<2 k} \leqslant H_{2}^{\prime<2 k} \\
& \quad=\operatorname{dim} H^{2}\left(G, H_{\text {odd }}^{<2 k}(X(\mathbb{C}), \mathbb{Z})\right)+\operatorname{dim} H^{1}\left(G, H_{\text {even }}^{<2 k}(X(\mathbb{C}), \mathbb{Z})\right) .
\end{aligned}
$$

Теорема 1.4 доказана. 
4. Замечания и примеры. Заметим, что при конкретном $k$ гомоморфизм

$$
\varrho_{k}: H_{\mathrm{alg}}^{k}(X(\mathbb{R}), \mathbb{Z} / 2) \rightarrow H_{\mathrm{alg}}^{2 k}(X(\mathbb{C}), \mathbb{Z} / 2),
$$

для которого коммутативна диаграмма, аналогичная диаграмме (0.3),

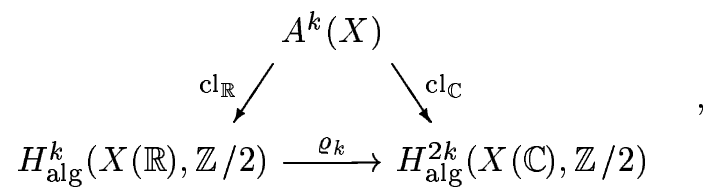

может быть определен и не только для $M$-многообразий. Например, всегда определен гомоморфизм

$$
\varrho_{0}: H_{\mathrm{alg}}^{0}(X(\mathbb{R}), \mathbb{Z} / 2) \rightarrow H_{\mathrm{alg}}^{0}(X(\mathbb{C}), \mathbb{Z} / 2),
$$

если $X(\mathbb{R}) \neq \varnothing$, так как

$$
H_{\text {alg }}^{0}(X(\mathbb{R}), \mathbb{Z} / 2)=\mathbb{Z} / 2 \cdot[X(\mathbb{R})]^{*}, \quad H_{\text {alg }}^{0}(X(\mathbb{C}), \mathbb{Z} / 2)=\mathbb{Z} / 2 \cdot[X(\mathbb{C})]^{*}
$$

и в силу диаграммы $(4.1)$ вьполняется равенство $\varrho_{0}\left([X(\mathbb{R})]^{*}\right)=[X(\mathbb{C})]^{*}$. Также, всегда определен гомоморфизм $\varrho_{n}$, где $n=\operatorname{dim} X$. Действительно, тогда

$$
\begin{aligned}
& H_{\text {alg }}^{n}(X(\mathbb{R}), \mathbb{Z} / 2)=H_{0}(X(\mathbb{R}), \mathbb{Z} / 2)=(\mathbb{Z} / 2)^{s}, \\
& H_{\text {alg }}^{2 n}(X(\mathbb{C}), \mathbb{Z} / 2)=H_{0}(X(\mathbb{C}), \mathbb{Z} / 2)=\mathbb{Z} / 2,
\end{aligned}
$$

где $s$ - число компонент связности множества $X(\mathbb{R})$, и в силу диаграммы (4.1) вьполняется равенство $\varrho_{n}\left(m_{1}, \ldots, m_{s}\right)=m_{1}+\cdots+m_{s}$. Если многообразие $X$ является $G M$-многообразием и инволюция комплексного сопряжения $g \in G(\mathbb{C} / \mathbb{R})$ действует на группе $H^{2 k}(X(\mathbb{C}), \mathbb{Z} / 2)$ тривиально, то гомоморфизм $\varrho_{k}$, для которого коммутативна диаграмма (4.1), также определен, так как в этом случае справедливо утверждение про $\operatorname{cl}_{\mathbb{R}}, \operatorname{cl}_{\mathbb{C}}(\mathrm{cm} .[1]):$ если $\mathrm{cl}_{\mathbb{R}}(Z)=0, \operatorname{mo~}_{\mathbb{C}}(Z)=0$. С другой стороны, например, для квадрики $X$, заданной уравнением

$$
x_{0}^{2}-x_{1}^{2}-x_{2}^{2}-x_{3}^{2}=0
$$

гомоморфизм

$$
\varrho_{1}: H_{\mathrm{alg}}^{1}(X(\mathbb{R}), \mathbb{Z} / 2) \rightarrow H_{\mathrm{alg}}^{2}(X(\mathbb{C}), \mathbb{Z} / 2),
$$

для которого коммутативна диаграмма (4.1), не определен, так как для кривой $Z \in$ $A^{1}(X)$, заданной уравнением $x_{3}=0$, вьполняется равенство $\mathrm{cl}_{\mathbb{R}}(Z)=0$ и неравенство $\operatorname{cl}_{\mathbb{C}}(Z) \neq 0$.

В общем случае определен гомоморфизм

$$
\varrho_{k}^{(\infty)}: H_{\mathrm{alg}}^{k}(X(\mathbb{R}), \mathbb{Z} / 2) \rightarrow H_{\infty}^{2 k}
$$

Тогда элемент $\varrho_{k}^{(\infty)}\left(\operatorname{cl}_{\mathbb{R}}(Z)\right)$ получается из элемента $\mathrm{cl}_{\mathbb{C}}(Z)$ с помошью факторизации. $\mathrm{C}$ другой стороны, этот гомоморфизм равен композиции гомоморфизмов

$$
H_{\mathrm{alg}}^{k}(X(\mathbb{R}), \mathbb{Z} / 2) \stackrel{S q}{\longrightarrow} \mathscr{F}^{2 k} \rightarrow \mathscr{F}^{2 k} / \mathscr{F}^{2 k-1} .
$$


В частности, если многообразие $X$ является $G M$-многообразием, то определен гомоморфизм

$$
\varrho_{k}^{(2)}: H_{\mathrm{alg}}^{k}(X(\mathbb{R}), \mathbb{Z} / 2) \rightarrow H_{2}^{2 k}=H^{2 k}(X(\mathbb{C}), \mathbb{Z} / 2)^{G} /\left(1+g^{*}\right) H^{2 k}(X(\mathbb{C}), \mathbb{Z} / 2) .
$$

В этом случае выполняются неравенства

$$
\begin{aligned}
\operatorname{dim} \operatorname{ker} \varrho_{k}^{(2)}+ & \operatorname{dim} H_{\mathrm{alg}}^{<k}(X(\mathbb{R}), \mathbb{Z} / 2) \leqslant \operatorname{dim} H^{1}\left(G, H^{<2 k}(X(\mathbb{C}), \mathbb{Z} / 2)\right), \\
& \operatorname{dim} H_{\mathrm{alg}}^{\leqslant k}(X(\mathbb{R}), \mathbb{Z} / 2) \leqslant \operatorname{dim} H^{1}\left(G, H^{\leqslant 2 k}(X(\mathbb{C}), \mathbb{Z} / 2)\right),
\end{aligned}
$$

впрочем, второе неравенство всегда вьполняется. Можно сформулировать аналогичные неравенства для $G M \mathbb{Z}$-многообразий.

\section{СПИСОК ЦИТИРОВАННОЙ ЛИТЕРАТУРЫ}

[1] Краснов В.А. Характеристические классы векторных расслоений на вещественном алгебраическом многообразии // Изв. АН СССР. Сер. матем. 1991. Т. 55. № 4. С. 716-747.

[2] Краснов В. А. Об эквивариантных когомологиях Гротендика вещественного алгебраического многообразия и их приложениях // Изв. РАН. Сер. матем. 1994. Т. 58. № 3. С. 36-52.

[3] Краснов В. А. Вещественные алгебраические $G M \mathbb{Z}$-поверхности // Изв. РАН. Сер. матем. 1998. T. 62. № 4. C. 51-80.

[4] Degtyarev A., Kharlamov V. Halves of real Enriques surface // Comment. Math. Helv. 1996. V. 71. P. 628-663.

[5] Калинин И.О. Когомологические характеристики вещественных проективных гиперповерхностей // Алгебра и анализ. 1991. Т. 3. № 2. С. 91-110.

[6] Краснов В.А. Неравенства Гарнака-Тома для отображений вещественных алгебраических многообразий // Изв. АН СССР. Сер. матем. 1983. Т. 47. № 2. С. 268-297.

[7] Краснов В.А. Вещественные алгебраические $G M$-многообразия // Изв. РАН. Сер. матем. 1998. T. 62. № 3. C. 39-66.

[8] Краснов В.А. Этальные и эквивариантные когомологии вещественного алгебраического многообразия // Изв. РАН. Сер. матем. 1998. Т. 62. №5. С. 166-186. 